\title{
X-RAY STUDY OF FERROELECTRIC PHASE TRANSITION IN $\left(\mathrm{NH}\left(\mathrm{CH}_{3}\right)_{3}\right)_{3} \mathrm{Sb}_{2} \mathrm{Cl}_{9}$
}

\author{
P.E. TomaszewsKi \\ Institute of Low Temperature and Structure Research, Polish Academy of Scicnces \\ P.O. Box 937, 50-950 Wrocław 2, Poland
}

(Received September 13, 1994; in final form November 28, 1994)

The lattice parameters of a title crystal, TMACA, were measured from a room temperature to $405 \mathrm{~K}$ by the $\mathrm{X}$-ray single-crystal Bond method. The high-temperature paraelectric phase should have the monoclinic symmetry (space group $P 2 / c$ ). Some peculiarities on dielectric behaviour of the crystal were explained. The hypotheses on incommensurability of the paraelectric phase as well as on the parent normal (trigonal) phase were presented.

PACS numbers: $61.50 . \mathrm{Ks}, 64.70 . \mathrm{Kb}, 65.70 .+\mathrm{y}, 77.80 . \mathrm{Bh}$

\section{Introduction}

Tris(trimethylammonium)nanochlorodiantimonate (III), $\left(\mathrm{NH}\left(\mathrm{CH}_{3}\right)_{3}\right)_{3} \mathrm{Sb}_{2} \mathrm{Cl}_{9}$ (abbreviated as TMACA), belongs to a rich family of compounds with the general formula $\mathrm{A}_{3} \mathrm{~B}_{2} \mathrm{X}_{9}$. More than 40 structural phase transitions have been observed in this type of crystals [1]. The structure of TMACA [2] contains $\mathrm{SbCl}_{6}$ bipyramids forming a two-dimensional network arranged in the elongated, left- and rightturned six-membered rings (described as the structure motif $A$ in Ref. [1]). Such description in term of isolated-layer structure is important to understand the phase transition mechanism. The TMACA crystal undergoes phase transitions from the paraelectric high-temperature phase to the ferroelectric one at about $365 \mathrm{~K}$ [3-6] and successively to the other phases at $203 \mathrm{~K}[4,6]$ and $125 \mathrm{~K}[7]$ with lowering temperature. The existence of an intermediate phase bctween 364 and $365 \mathrm{~K}$ was supposed on the basis of dielectric measurements [6].

Our studies concerned the high-temperature region with the aim to describe the structural changes during the phase transition; the supposed intermediate phase was out of scope of this paper.

\section{Experimental}

The TMACA samples used in this study were the same as in the dielectric measurements published separately [5]. The X-ray powder diffraction photographs were made with temperature by using a high-temperature Guinier camera. 
The lattice parameter measurements were carried out from a room temperature to $405 \mathrm{~K}$ by the $\mathrm{X}$-ray single-crystal Bond diffractometer $[8,9]$. However, the quality of a crystal and a high level of absorption allow us only to measure the Bragg angles for rather low-angle reflections (10 $000,0100,0016,4012$, $8014,1108,10012,1204)$ by using $\mathrm{Cu} K_{\alpha_{1}}$ radiation $(\lambda=0.1540562 \mathrm{~nm})$. Thus, the relative accuracy $\Delta d / d$ of lattice parameter measurements is of about $10^{-5}$. Covering of TMACA crystals by silicon grease was necessary to prevent a partial (mainly on surface) decomposition of the sample with time and increasing temperature.

\section{Results and discussion}

The Guinier powder photographs indicate the structural phase transition at $365 \mathrm{~K}$ and the decomposition of the sample at about $415 \mathrm{~K}$.

The lattice parameters $a, b, c$ and $\beta$, calculated from $\theta$-values of reflections are plotted as a function of temperature in Fig. 1. The values for the room temperature ferroelectric phase, $a=1.0075, b=0.9076, c=1.5460 \mathrm{~nm}$ and $\beta=89.79^{\circ}$ (at $296 \mathrm{~K}$ ) are in agreement with the literature data [2]. Only the monoclinic angle $\beta$ was chosen as acute-one. It should be noted that the thermal expansion of the polar $c$-axis is three times higher than for the other axes.

The very interesting result was obtained for thermal dependence of monoclinic angle $\beta$. It goes from acute angle below about $330 \mathrm{~K}$ to obtuse-one reaching a temperature independent value of about $91^{\circ}$ above the transition. These changes of $\beta$-angle correspond to gliding of layers in the structure along the polar axis $c$. Thus, the reversion of spontaneous polarization (connected with the change of domain orientation from $-\beta$ to $+\beta$ ) is more easy to obtain when the monoclinic angle $\beta$ is closer to $90^{\circ}$, i.e. at about $330 \mathrm{~K}$. This might explain the difficulties in the observation of dielectric hysteresis loop at room temperature [5].

Conserving the general motif $A$ at the transition one can consider the high-temperature paraelectric phase as a kind of average structure of both left- and right-turned motives (Fig. 2) realized as a statistical distribution of microdomains with the opposite polarization. The phase transition should be then of order-disorder type. Due to the conservation of a monoclinic angle the resulting symmetry should be $P 2 / c$ while in the ferroelectric phase the symmetry is $P c$ [2]. The microdomain structure leads to an enlarging of Bragg reflection width and poor quality of the diffraction pattern. This does not allow us to perform a routine crystal structure analysis.

The temperature dependence of dielectric permittivity with a minimum at about $385 \mathrm{~K}[10]$ and the peculiar kind of the thermal hysteresis $[6,10]$ are characteristic for the transition from an incommensurate to a commensurate phase like in $\mathrm{Rb}_{2} \mathrm{ZnCl}_{4}$ [11-14], $\mathrm{Rb}_{2} \mathrm{ZnBr}_{4}$ [14], $\left(\mathrm{NH}_{4}\right)_{2} \mathrm{BeF}_{4}$ [15] and $\mathrm{SC}\left(\mathrm{ND}_{2}\right)_{2}$ [16], where such behaviour has been attributed to the pinning of the incommensurate waves by impurities or defects in the crystal. It seems that the above-mentioned averaging of two orientations in high-temperature phase may be a source of such behaviour. Unfortunately, the experimental verification (search for X-ray satellite reflections) for TMACA seems to be difficult to perform and was out of scope of this paper. But in any case the replacement of both phase transitions at about 


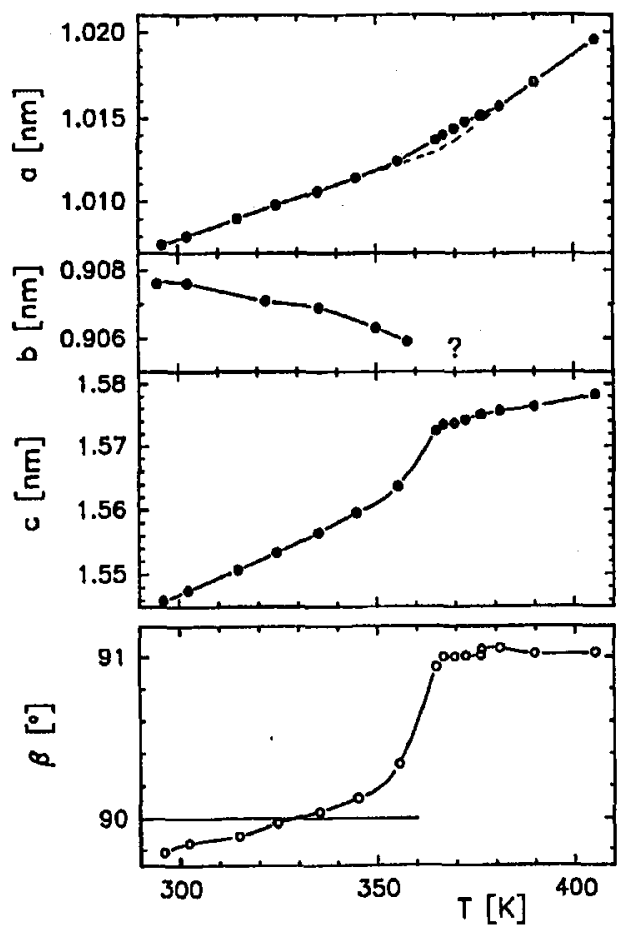

Fig. 1. Temperature dependence of the lattice parameters of TMACA. The sign "?" corresponds to the lack of data at the high-temperature phase.

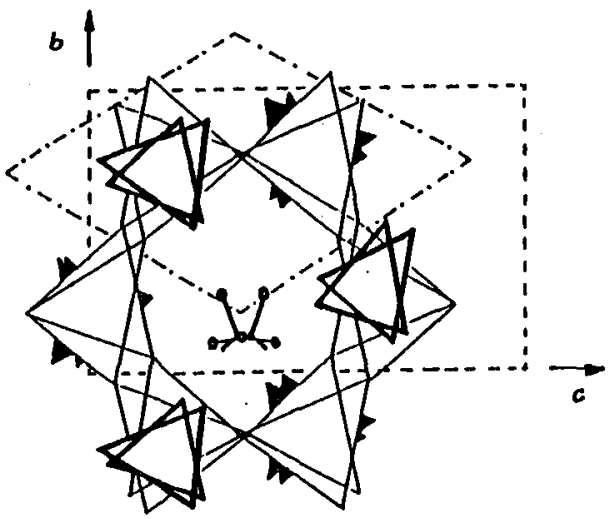

Fig. 2. Schematic drawing of the TMACA structure in the paraelectric phase. The vertices of each triangle correspond to $\mathrm{Cl}$-atoms. The bold and fine lines correspond to two levels in the structure viewed along $a$-axis (pseudohexagonal); solid triangles represent the lowest level in the structure of $\mathrm{SbCl}_{9}$-layer. Dashed line represents the unit cell; dashed-pointed line - a unit cell of a hypothetical trigonal phase. 
$365 \mathrm{~K}$ (inverse sequence on cooling and heating) supposed in Ref. [6] is not correct and should be rejected. However, the anomalous region of lattice parameters temperature behaviour corresponds well to this thermal "hysteresis" on dielectric permittivity.

This hypothesis of the incommensurate phase transition implies the existence of a normal phase (real or hypothetical, with the trigonal symmetry) and consequently the phase transition from the normal to the incommensurate phase with the multiplication of a unit cell. TMACA, however, is decomposed before this transition takes place. Perhaps such a phase could be reached under the high pressure; a preliminary optical examination shows the appearing of the trigonal domain structure after applying the pressure [10].

\section{Acknowledgments}

The author would like to thank Prof. Z. Czapla, Drs. R. Cach and A. Mikolajczak from the Institute of Experimental Physics, Wrocław University, for supplying with TMACA crystals and for presenting unpublished data of dielectric and optical experiments and for stimulating discussions. I also thank Dr. D. Kucharczyk for helpful discussions during the Bond experiment and Prof. A. Pietraszko for making the Guinier photographs (both from our Institute).

\section{References}

[1] P.E. Tomaszewski, Phys. Stat. Solidi B 181, 15 (1994).

[2] A. Kallel, J.W. Bats, Acta Crystallogr. C 41, 1022 (1985).

[3] R. Jakubas, Z. Czapla, Z. Galewski, L. Sobczyk, Ferroelectrics Lett. 5, 143 (1986).

[4] J. Mróz, Z. Czapla, R. Jakubas, Acta Phys. Pol. A 70, 705 (1986).

[5] A. Mikolajczak, R. Cach, Z. Czapla, R. Jakubas, Acta Phys. Pol. A 74, 417 (1988).

[6] A. Miniewicz, J. Sworakowski, R. Jakubas, M. Bertault, C. Ecolivet, Ferroelectrics 94, 323 (1989).

[7] S. Idziak, R. Jakubas, Solid State Commun. 62, 173 (1987).

[8] W.L. Bond, Acta Crystallogr. 13, 814 (1960); ibid. A 31, 698 (1975).

[9] K. Łukaszewicz, D. Kucharczyk, M. Malinowski, A. Pietraszko, Kristall u. Technik 13, 561 (1978).

[10] R. Cach, A. Mikolajczak, private communication.

[11] K. Hamano, K. Ema, S. Hirotsu, Ferroelectrics 36, 343 (1981).

[12] K. Hamano, Y. Ikeda, T. Fujimoto, K. Ema, S. Hirotsu, J. Phys. Soc. Jpn. 49, 2278 (1980).

[13] K. Deguchi, Y. Okada, H. Fukunaga, E. Nakamura, J. Phys. Soc. Jpn. 56, 208 (1987).

[14] K. Ilamano, T. Hoshinuma, K. Ema, J. Phys. Soc. Jpn. 50, 2666 (1981).

[15] B.A. Strukov, V.M. Arutyanova, I. Uesi, Fiz. Tverd. Tela 24, 3061 (1982).

[16] G. Andre, D. Durand, F. Denoyer, R. Currat, F. Moussa, Phys. Rev. B 35, 2909 (1987). 